Supporting Information

\title{
Photo-induced Crawling Motion of Azobenzene Crystals on Modified Gold Surfaces
}

\author{
$\underline{\text { Yasuo Norikane }}^{* 1,2}$, Masaru Hayashino ${ }^{1,3}$, Mio Ohnuma ${ }^{1}$, Koji Abe ${ }^{1}$, Yoshihiro \\ Kikkawa $^{1}$, Koichiro Saito ${ }^{1}$, Kengo Manabe ${ }^{1}$, Koji Miyake ${ }^{4}$, Miki Nakano ${ }^{4}$, Naoki \\ Takada $^{5}$
}

1 Research Institute for Advanced Electronics and Photonics, National Institute of Advanced Industrial Science and Technology (AIST), Tsukuba, Ibaraki, 305-8565, Japan 2 Department of Chemistry, Faculty of Pure and Applied Sciences, University of Tsukuba, Ibaraki, 305-8571, Japan

3 Graduate School of Pure and Applied Sciences, University of Tsukuba, Ibaraki, 3058571, Japan

4 Advanced Manufacturing Research Institute, National Institute of Advanced Industrial Science and Technology (AIST), Tsukuba, Ibaraki, 305-8564, Japan

5 Research Institute for Energy Conservation, National Institute of Advanced Industrial Science and Technology (AIST), Tsukuba, Ibaraki, 305-8564, Japan

Email:y-norikane@aist.go.jp 


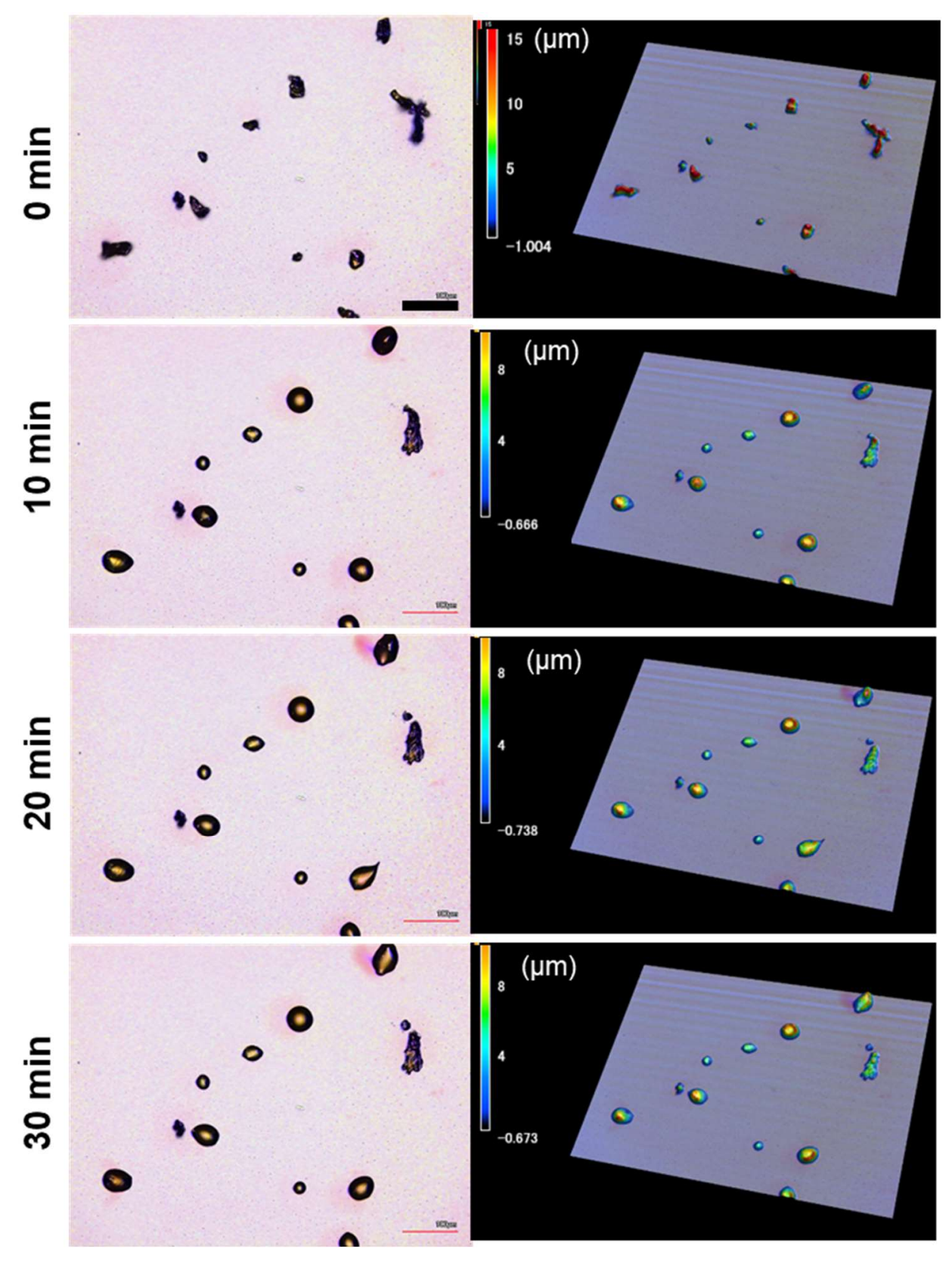

Figure S1 Photomicrographs of DMAB crystals on HDTMS modified glass surface after irradiation for $\mathrm{t}=0,10,20$ and $30 \mathrm{~min}$. The left column is photomicrographs of the entire field of view, and the right column is the $3 \mathrm{D}$ images obtained by a laser scanning microscope. Light irradiation was performed from the left for UV (365 nm) and from the right for visible (465 nm) light. Scale bar: $100 \mu \mathrm{m}$. 
Table S1 Dynamic Contact Angles and Hystereses of Water on Selected Surfaces

\begin{tabular}{|l|c|c|c|} 
& $\theta_{A d v}[$ degree $]$ & $\theta_{\text {Rec }}[$ degree $]$ & $\cos \theta_{\text {Rec }}-\cos \theta_{A d v}$ \\
\hline $\begin{array}{l}\text { HDTMS modified } \\
\text { glass }\end{array}$ & 103.0 & 74.1 & 0.50 \\
\hline $\mathrm{C}_{16} \mathrm{SH}$ modified gold & 116.3 & 94.4 & 0.37 \\
\hline
\end{tabular}
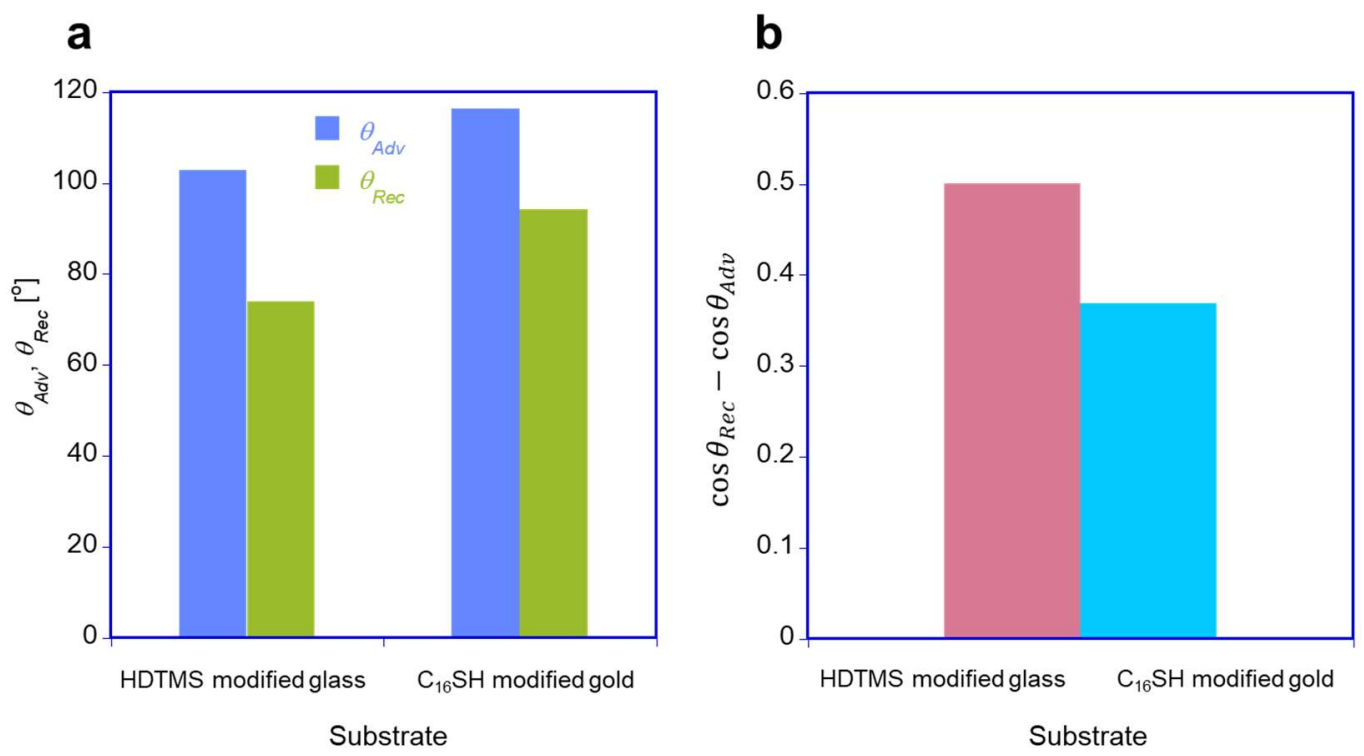

Figure S2 Surface wettability of selected surfaces. (a) Advancing contact angles $\left(\theta_{\text {Adv }}\right)$ and receding contact angles $\left(\theta_{R e c}\right)$ of water droplet on each surface for calculating (b) contact angle hysteresis $\left(\cos \theta_{\text {Rec }}-\cos \theta_{A d v}\right)$. 

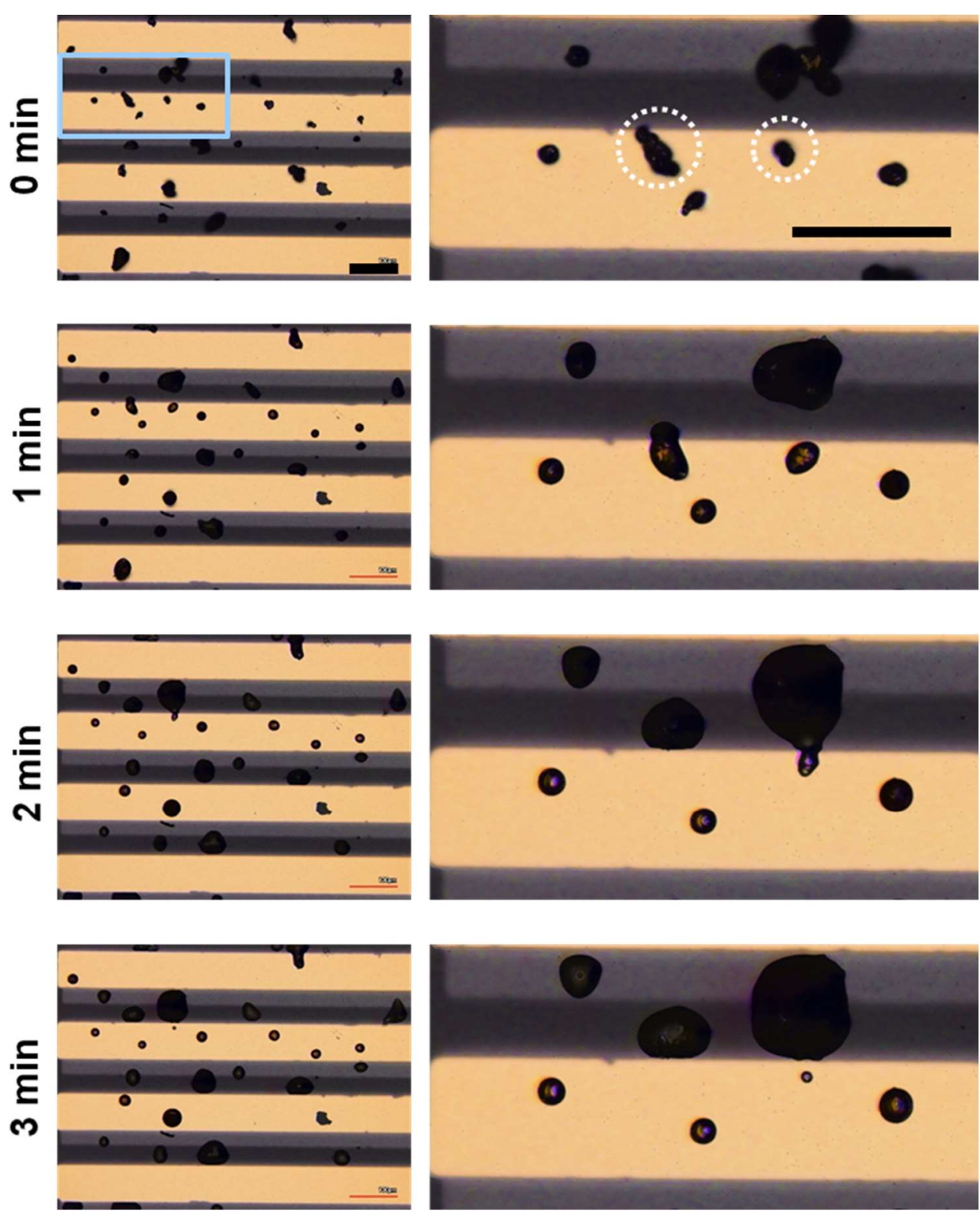

Figure S3 Photomicrographs for the crawling motion of DMAB crystals on alternating $\mathrm{C}_{16} \mathrm{SH}$ modified gold / HDTMS modified glass surface after irradiation for $\mathrm{t}=0,1,2$ and $3 \mathrm{~min}$. The left column is photomicrographs of the entire field of view, and the right column is the enlarged image of the square in the first column. Crystals in dashed circles were attracted to the glass region. Light irradiation was performed from the left for UV $(365 \mathrm{~nm})$ and from the right for visible (465 nm) light. Scale bar: $100 \mu \mathrm{m}$. 